\title{
Continuous-time quantum walk on graph associated with quantum Bernoulli noises
}

\author{
Yanyan Wang, Caishi Wang, Yan Luo, Guangbo Yang
}

\begin{abstract}
In this paper, we introduce a kind of graph relations based on quantum Bernoulli noises and investigate the corresponding continuous-time quantum walks on these graphs. Evolution properties are examined of the walks and first step results are obtained.
\end{abstract}

\section{Keywords - Quantum Bernoulli noise, graph relation, continuous-time quantum walk}

\section{INTRODUCTION}

In recent years, quantum walks have been intensively investigated, with the hope that they may be useful in constructing new efficient quantum algorithms (see [1-3] for reviews of quantum walks). Quantum walks can be viewed as quantum analogs of the classical random walks. Whereas, studies have shown that quantum walks are distinct from their classical counterparts [12]. There are two different types of quantum walks: discrete-time ones and continuous-time ones. Here we focus only on continuous-time ones.

Continuous-time quantum walks (CTQWs) were introduced by Farhi and Gutmann [12] as a quantum-mechanical transport process on discrete structure, generally called graphs. For the past few years, CTQWs have been considered on diverse graphs such as integer lattice [14-16], distance regular graphs [17], $n$-cube [18], start-graphs [19-20], Apolloman network [17] and circulantBunkeds [22].

Quantum Bernoulli noises [10] are annihilation and creation operators acting on Bernoulli functionals, which satisfy the canonical anti-commutation relations (CAR) in equal time. It turns out that quantum Bernoulli noises can play

Let $L^{2}(Z)$ be the space of square integrable complex-valued random variables on $(\Omega, \mathcal{F}, \mathrm{P})$. It is known that $\mathrm{L}^{2}(\mathrm{Z})$ has an orthonormal basis of form $\left\{\mathrm{Z}_{\tau} \mid \tau \in \Gamma\right.$, where $\mathrm{Z}_{\emptyset}=1$ and $\mathrm{Z}_{\tau}=\prod_{\mathrm{i} \in \tau} \mathrm{Z}_{\mathrm{i}}$ for $\tau \in \Gamma, \tau \neq \emptyset$ an important role in the study of quantum Markov semigroups and stochastic Schrodinger equations [11]. Recently, Wang and Ye [9] have constructed a model of discrete-time quantum walk in terms of quantum Bernoulli noise and shown its interesting properties.

In this paper, we introduce a graph relation associated with quantum Bernoulli noises and construct a model of CTQW based on the graph relation. We examine properties of the model and obtain several interesting results. The paper is organized as follows. In Section 2, we describe the graph relation and define the model. Properties of the model are shown in Section 3.

\section{II.GRAPH RELATION}

In this section, we first briefly recall some notions and facts about quantum Bernoulli noises [9]. And then, we introduce a graph relation associated with quantum Bernoulli noises.

Let $\mathbb{N}$ be the set of all nonnegative integers and $\Gamma$ the finite power set of $\mathbb{N}$, namely

$$
\Gamma=\{\tau \mid \tau \subset \mathbb{N} \text { and } \# \tau<\infty\},
$$

where \# $\pi$ denotes the cardinality of $\tau$ as a set. Throughout this section, we suppose that $(\Omega, \mathcal{F}, \mathrm{P})$ is a probability space and $Z=\left\{Z_{n}\right\}_{n \geq 0}$ is an independent sequence of random variables on $(\Omega, \mathcal{F}, \mathrm{P})$, which satisfies that:

$$
\mathrm{P}\left\{\mathrm{Z}_{\mathrm{n}}=\theta_{\mathrm{n}}\right\}=\mathrm{p}_{\mathrm{n}}, \quad \mathrm{P}\left\{\mathrm{Z}_{\mathrm{n}}=-1 / \theta_{\mathrm{n}}\right\}=\mathrm{q}_{\mathrm{n}},
$$

where $\theta_{\mathrm{n}}=\sqrt{\mathrm{q}_{\mathrm{n}} / \mathrm{p}_{\mathrm{n}}}, 0<\mathrm{p}_{\mathrm{n}}<1$. We denote by $\mathcal{F}=$ $\sigma\left\{Z_{n}, n \geq 0\right\}$ the $\sigma$-field generated by $Z=\left\{Z_{n}\right\}_{n \geq 0}$.

Lemma 1. [10] For $\mathrm{k} \geq 0$, there exists a bounded operator $\partial_{\mathrm{k}}$ on $\mathrm{L}^{2}(\mathrm{Z})$ such that

$$
\partial_{\mathrm{k}} \mathrm{Z}_{\tau}=\chi_{\tau}(\mathrm{k}) \mathrm{Z}_{\tau \backslash \mathrm{k}}, \quad \tau \in \Gamma,
$$


where $\tau \backslash \mathrm{k}=\tau \backslash\{\mathrm{k}\}$, and $\chi_{\tau}(\mathrm{k})$ is the indicator of $\tau$ as a subset of $\mathbb{N}$.

$$
\partial_{\mathrm{k}}^{*}=\left(1-\chi_{\tau}(\mathrm{k})\right) \mathrm{Z}_{\tau \cup \mathrm{k}}, \quad \tau \in \Gamma,
$$

where $\tau \cup \mathrm{k}=\tau \cup\{\mathrm{k}\}$.

We note that the sequence $\left\{\partial_{\mathrm{k}}, \partial_{\mathrm{k}}^{*}\right\}_{\mathrm{k} \geq 0}$ is known as quantum Bernoulli noise (QBN, for short).

Let $\mathrm{N} \in \mathbb{N}$ be a fixed nonnegative integer and $\Gamma_{\mathrm{N}}$ the power set of $\{0,1,2,3, \cdots, N\}$, namely

$$
\Gamma_{\mathrm{N}}=\{\sigma \mid \sigma \subset\{0,1,2,3, \cdots, \mathrm{N}\}\} .
$$

It is obviously that $\Gamma_{\mathrm{N}}$ just has $2^{\mathrm{N}+1}$ elements . For $\sigma, \tau \in \Gamma_{\mathrm{N}}$ with $\sigma \neq \tau$, we use $\sigma<\tau$ to mean one of the following two relations holds true:

Relation $1 . \# \sigma<\# \tau$, where \# $\sigma$ and $\# \tau$ denote the
Lemma 2. [10] For $\mathrm{k} \geq 0$, the adjont operator $\partial_{\mathrm{k}}^{*}$ of $\partial_{\mathrm{k}}$ has following property:

cardinalities of $\sigma$ and $\tau$, respectively;

Relation 2. \# $\sigma=\# \tau$, but

$10^{\mathrm{k}-1} \mathrm{i}_{1}+10^{\mathrm{k}-2} \mathrm{i}_{2}+\cdots+10^{0} \mathrm{i}_{\mathrm{k}}<10^{\mathrm{k}-1} \mathrm{j}_{1}+$ $10^{\mathrm{k}-2} \mathrm{j}_{2}+\cdots+10^{0} \mathrm{j}_{\mathrm{k}}$

where $\sigma=\left\{\mathrm{i}_{1}, \mathrm{i}_{2}, \cdots, \mathrm{i}_{\mathrm{k}}\right\}$ with $\mathrm{i}_{1}<\mathrm{i}_{2}<\cdots<\mathrm{i}_{\mathrm{k}} ，$ $\tau=\left\{\mathrm{j}_{1}, \mathrm{j}_{2}, \cdots, \mathrm{j}_{\mathrm{k}}\right\}$ with $\mathrm{j}_{1}<\mathrm{j}_{2}<\cdots<\mathrm{j}_{\mathrm{k}}$ and $\mathrm{k}=\# \sigma=\# \tau$.

It can be seen that for $\sigma, \tau \in \Gamma_{\mathrm{N}}$ with $\sigma \neq \tau$ one has $\sigma<\tau$ or $\tau<\sigma$, which actually defines an order relation in $\Gamma_{\mathrm{N}}$. By using this order relation, we can enumerate elements of $\Gamma_{\mathrm{N}}$ as follows

$$
\Gamma_{\mathrm{N}}=\left\{\sigma_{1}, \sigma_{2}, \cdots, \sigma_{2} \mathrm{~N}+1\right\}
$$

with $\sigma_{1}<\sigma_{2}<\cdots<\sigma_{2^{N+1}}$. The following table shows the cases for $\mathrm{N}=0,1$ and 2 .

\begin{tabular}{c|c|c|c}
\hline$\sigma^{N}$ & $\mathrm{~N}=0$ & $\mathrm{~N}=1$ & $\mathrm{~N}=2$ \\
\hline$\sigma_{1}$ & $\emptyset$ & $\emptyset$ & $\emptyset$ \\
\hline$\sigma_{2}$ & $\{0\}$ & $\{0\}$ & $\{0\}$ \\
\hline$\sigma_{3}$ & & $\{1\}$ & $\{1\}$ \\
\hline$\sigma_{4}$ & & $\{0,1\}$ & $\{2\}$ \\
\hline$\sigma_{5}$ & & & $\{0,1\}$ \\
\hline$\sigma_{6}$ & & & $\{1,2\}$ \\
\hline$\sigma_{7}$ & & & $\{1,2,3\}$ \\
\hline$\sigma_{8}$ & & & \\
\hline
\end{tabular}

Table 1.

We now introduce a graph relation " $\sim "$ in $\Gamma_{\mathrm{N}}$ as follows. Two elements $\sigma_{\mathrm{i}}, \sigma_{\mathrm{j}}$ of $\Gamma_{\mathrm{N}}$ are said to form an edge, written as $\sigma_{\mathrm{i}} \sim \sigma_{\mathrm{j}}$, if there exists a unique $\mathrm{k} \in\{0,1,2,3, \cdots, \mathrm{N}\}$ such that

$\partial_{\mathrm{k}} \mathrm{Z}_{\sigma_{\mathrm{i}}}=\mathrm{Z}_{\sigma_{\mathrm{j}}}$ or $\partial_{\mathrm{k}}^{*} \mathrm{Z}_{\sigma_{\mathrm{i}}}=\mathrm{Z}_{\sigma_{\mathrm{j}}}$.

Let $\mathrm{E}$ be the set of edges defined by the above graph relation. Then we come to a graph

$$
\mathrm{G}=(\mathrm{V}, \mathrm{E})
$$

with $\mathrm{V}=\Gamma_{\mathrm{N}}$, which is called the graph of order $\mathrm{N}$ associated with quantum Bernoulli noises. The adjacent matrix $A$ of $G$ is given by

$$
A_{i j}=\left\{\begin{array}{cc}
1, & \text { if } \sigma_{i} \sim \sigma_{j} \\
0, & \text { others. }
\end{array}\right.
$$

It is easy to see that the graph $G=(V, E)$ defined above is symmetric. Thus its Hamiltonian $H_{N}=D_{N}-A_{N}$ is Hermitian, where $D_{N}$ is a diagonal matrix with its $i$ th diagonal entry $\mathrm{D}_{\mathrm{N}_{\mathrm{ii}}}=\operatorname{deg}\left(\sigma_{\mathrm{i}}\right)$, namely, $\mathrm{D}_{\mathrm{N}_{\mathrm{ii}}}$ is the degree of vertex $\sigma_{\mathrm{i}}$. By lengthy but straightforward calculations, we can work out the matrices $D_{N}, A_{N}$ and $H_{N}$ of graph $G$ for $\mathrm{N}=0,1$ and 2 .

Example 1. For $\mathrm{N}=0$, the corresponding matrices $D_{0}, A_{0}$ and $H_{0}$ of graph $G$ have the following forms 


$$
\mathrm{D}_{0}=\left(\begin{array}{ll}
1 & 0 \\
0 & 1
\end{array}\right), \mathrm{A}_{0}=\left(\begin{array}{ll}
0 & 1 \\
1 & 0
\end{array}\right), \mathrm{H}_{0}=\left(\begin{array}{cc}
1 & -1 \\
-1 & 1
\end{array}\right) .
$$

Example 2. For $\mathrm{N}=1$, the corresponding matrices $\mathrm{D}_{1}, \mathrm{~A}_{1}$ and $\mathrm{H}_{1}$ of graph $\mathrm{G}$ take the following forms

$$
\mathrm{D}_{1}=\left(\begin{array}{llll}
2 & 0 & 0 & 0 \\
0 & 2 & 0 & 0 \\
0 & 0 & 2 & 0 \\
0 & 0 & 0 & 2
\end{array}\right), \mathrm{A}_{1}=\left(\begin{array}{llll}
0 & 1 & 1 & 0 \\
1 & 0 & 0 & 1 \\
1 & 0 & 0 & 1 \\
0 & 1 & 1 & 0
\end{array}\right), \mathrm{H}_{1}=\left(\begin{array}{cccc}
2 & -1 & -1 & 0 \\
-1 & 2 & 0 & -1 \\
-1 & 0 & 2 & -1 \\
0 & -1 & -1 & 2
\end{array}\right) .
$$

Example 3. For $\mathrm{N}=2$, the corresponding matrices $\mathrm{D}_{2}, \mathrm{~A}_{2}$ and $\mathrm{H}_{2}$ of graph $\mathrm{G}$ are of the following forms

$$
\mathrm{D}_{2}=\left(\begin{array}{llllllll}
3 & 0 & 0 & 0 & 0 & 0 & 0 & 0 \\
0 & 3 & 0 & 0 & 0 & 0 & 0 & 0 \\
0 & 0 & 3 & 0 & 0 & 0 & 0 & 0 \\
0 & 0 & 0 & 3 & 0 & 0 & 0 & 0 \\
0 & 0 & 0 & 0 & 3 & 0 & 0 & 0 \\
0 & 0 & 0 & 0 & 0 & 3 & 0 & 0 \\
0 & 0 & 0 & 0 & 0 & 0 & 3 & 0 \\
0 & 0 & 0 & 0 & 0 & 0 & 0 & 3
\end{array}\right), A_{2}=\left(\begin{array}{llllllll}
0 & 1 & 1 & 1 & 0 & 0 & 0 & 0 \\
1 & 0 & 0 & 0 & 1 & 1 & 0 & 0 \\
1 & 0 & 0 & 0 & 1 & 0 & 1 & 0 \\
1 & 0 & 0 & 0 & 0 & 1 & 1 & 0 \\
0 & 1 & 1 & 0 & 0 & 0 & 0 & 1 \\
0 & 1 & 0 & 1 & 0 & 0 & 0 & 1 \\
0 & 0 & 1 & 1 & 0 & 0 & 0 & 1 \\
0 & 0 & 0 & 0 & 1 & 1 & 1 & 0
\end{array}\right),(9)
$$

and $\mathrm{H}_{2}=\mathrm{D}_{2}-\mathrm{A}_{2}$.

\begin{tabular}{|c|c|c|}
\hline & $\lambda_{\mathrm{n}}$ & $\epsilon_{\mathrm{n}}$ \\
\hline $\mathrm{H}_{0}$ & $\lambda_{0}=0, \lambda_{1}=2$ & $\begin{aligned} \epsilon_{0} & =\frac{1}{\sqrt{2}}(1,1)^{\mathrm{T}} \\
\epsilon_{1} & =\frac{1}{\sqrt{2}}(-1,1)^{\mathrm{T}}\end{aligned}$ \\
\hline $\mathrm{H}_{1}$ & $\lambda_{0}=0, \lambda_{1}=\lambda_{2}=2, \lambda_{3}=4$ & $\begin{array}{c}\epsilon_{0}=\frac{1}{\sqrt{4}}(1,1,1,1)^{\mathrm{T}} \\
\epsilon_{1}=\frac{1}{\sqrt{4}}(1,-1,1,-1)^{\mathrm{T}} \\
\epsilon_{1}=\frac{1}{\sqrt{4}}(1,1,-1,-1)^{\mathrm{T}} \\
\epsilon_{2}=\frac{1}{\sqrt{4}}(1,-1,-1,1)^{\mathrm{T}}\end{array}$ \\
\hline $\mathrm{H}_{2}$ & $\begin{array}{c}\lambda_{0}=0 \\
\lambda_{1}=\lambda_{2}=\lambda_{3}=2, \lambda_{4}=\lambda_{5}=\lambda_{6}=4 \\
\lambda_{7}=6\end{array}$ & $\ldots$ \\
\hline
\end{tabular}

Proposition 1. For $\mathrm{N}=0,1$ and 2, the eigenvalues and the corresponding eigenvectors of Hamiltonian $\mathrm{H}_{\mathrm{N}}$ are as follows

Table 2. Eigenvalues $\lambda_{n}$ and eigenvectors $\epsilon_{n}$ of $H_{N}$.

\section{Continuous-time quantum walk}

In the present section, we investigate the continuous-time quantum walk on the graph $G=(V, E)$ introduced in the previous section.

Recall that $V=\Gamma_{N}$, which exactly contains $2^{\mathrm{N}+1}$ elements. In view of this, we can take the canonical complex Hilbert space $\mathcal{H}$ of dimension $2^{\mathrm{N}+1}$ as the state space of the walk that we will consider below. By convention, the inner product $\lessdot, \gg$ of $\mathcal{H}$ is linear in its second variable and conjugate linear in its the first variable. Let

$$
\left\{\omega_{j} \mid i=1,2,3, \cdots, 2^{N+1}\right\}
$$

be the canonical orthonormal basis for $\mathcal{H}$. Then, the Hamiltonian $\mathrm{H}_{\mathrm{N}}$ of $\mathrm{G}$ acts just as an Hermitian operator on $\mathcal{H}$ in a natural way.

Definition 1. The evolution equation of the continuous-time quantum walk on the graph $G$ takes the following form

$$
\mathrm{i} \frac{\mathrm{d}}{\mathrm{dt}} \varphi(\mathrm{t})=\mathrm{H}_{\mathrm{N}} \varphi(\mathrm{t})
$$

where $H_{N}$ is the Hamiltonian of the graph and $\varphi(t)$ 
denotes the state of the walk at time $t \geq 0$, which is a unit vector in $\mathcal{H}$. The probability $\mathrm{P}(\mathrm{t})$ of the walk at vertex $\sigma_{\mathrm{j}}$ and time $t \geq 0$ is given by $\mathrm{P}_{\mathrm{j}}(\mathrm{t})=\left|<\omega_{\mathrm{j}}, \varphi(\mathrm{t})>\right|^{2}$, where $\omega_{\mathrm{j}}$ is the basis vector of the canonical orthonormal basis for $\mathcal{H}$.

$$
\varphi(\mathrm{t})=\sum_{\mathrm{n}=0}^{2^{\mathrm{N}+1}} \mathrm{e}^{-\mathrm{i} t \lambda_{\mathrm{n}}}\left|\epsilon_{\mathrm{n}}><\epsilon_{\mathrm{n}}\right| \varphi(0),
$$

where $\lambda_{\mathrm{n}}$ is the eigenvalue of $\mathrm{H}_{\mathrm{N}}$ and $\epsilon_{\mathrm{n}}$ the corresponding eigenvector. By using this representation, we can get a formula for calculating the probability of the walk at vertex $\sigma_{\mathrm{j}}$ and time $\mathrm{t} \geq 0$, which reads

$$
\mathrm{P}_{\mathrm{j}}(\mathrm{t})=\left|\sum_{\mathrm{n}=0}^{2^{\mathrm{N}+1}} \mathrm{e}^{-\mathrm{i} t \lambda_{\mathrm{n}}}<\epsilon_{\mathrm{n}}, \varphi(0)><\omega_{\mathrm{j}}, \epsilon_{\mathrm{n}}>\right|^{2} .
$$

The average probability $\overline{\mathrm{P}_{\mathrm{j}}}$ of the walk at vertex $\sigma_{\mathrm{j}}$ is defined as

$$
\overline{P_{j}}=\lim _{T \rightarrow \infty} \frac{\int_{0}^{T} P_{j}(t) d t}{T} .
$$

Applying the above formula to the case of $\mathrm{N}=0$,

Proposition 2 . Let $\mathrm{N}=1$. Then at time $\mathrm{t} \geq 0$ the walk has a probability distribution given by

$$
\begin{aligned}
& \mathrm{P}_{1}(\mathrm{t})=\frac{6}{16}+\frac{4}{16} \cos 2 \mathrm{t}+\frac{2}{16} \cos 4 t+\frac{4}{16} \cos 2 \mathrm{t} \cos 4 t+\frac{4}{16} \sin 2 \mathrm{t} \sin 4 \mathrm{t} \\
& \mathrm{P}_{2}(\mathrm{t})=\frac{2}{16}-\frac{2}{16} \cos 4 \mathrm{t} \\
& \mathrm{P}_{3}(\mathrm{t})=\frac{2}{16}-\frac{2}{16} \cos 4 \mathrm{t} \\
& \mathrm{P}_{4}(\mathrm{t})=\frac{6}{16}-\frac{4}{16} \cos 2 \mathrm{t}+\frac{2}{16} \cos 4 \mathrm{t}-\frac{4}{16} \cos 2 \mathrm{t} \cos 4 \mathrm{t}-\frac{4}{16} \sin 2 \mathrm{t} \sin 4 \mathrm{t}
\end{aligned}
$$

Here the walk is assumed to start at vertex $\sigma_{1}$.

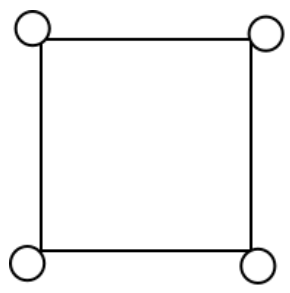

Fig 1. the graph in the case $\mathrm{N}=1$

\section{ACKNOWLEDGEMENT}

This work is supported by National Science Foundation of China (Grant No.11461061 and 11861057).

\section{REFERENCES}

[1] A. Ambainis, Int. J. Quantum Inf. 1, 507 (2003).

[2] J. Kemp, Contemp. Phsy. 44, 307 (2003).

[3] B. Tregenna, W. Flanagan, W. Maile, and V. Kendon, New J. Phys. 5, 83 (2003).

[4] N. Konno, J. Quantum Inf. Process. 1, 345 (2002).

[5] D. A. Meyer, J. Stat. Phys. 85, 551 (1996).

[6] D. Aharonov, A. Ambainis, J. Kempe. and U. V. Vazirani,
Proceedings of the 33rd Annual ACM Symposium on Theory of Computing (ACM Press, New York, 2001), p. 37.

[7] M. Hamada, N. Konno, and E. Segawa, RIMS Kokyouroku, No.1442, 1 (2005); e-print quant-ph/0408100.

[8] A. Romaneli, A. C. SicardiSchifino, R. Siri, G. Abal, A. Auyuanet, and R. Donangelo, Physica A 338, 395 (2004).

[9] C. Wang, and X. Ye . "Quantum walk in terms of quantum Bernoulli noises." Quantum Information Processing 15, 5:1-12, (2016).

[10] C. Wang, H. Chai, and Y. Lu, Discrete-time quantum Bernouli noise, J. Math. Phys. 5, 51, 053528 (2010).

[11] C. Wang and J. Chen, Quantum Markov semigroups constructed from quantum Bernoulli noise, J. Math.Phys. 2, 57, 023502 (2016).

[12] A. M. Childs, E. Farhi, and S. Gutmanna, Quantum Inf. Process.1, 35 (2002).

[13] E. Farhi and S. Gutmann, Phys. Rev. A 58, 915 (1998).

[14] N. Inui, K. Kasahara, Y. Konishi, and N. Konno, Fluct. Noise Lett. 5, 73 (2005).

[15] M. A. Jafarizadeh and S. Salimi, Ann. Phys. 322, 1005 (2007).

[16] M. A. Jafarizadeh, R. Sufiani, S. Salimi and s. Jafarizadeh, Eur. Phys. J. B 59, 199 (2007).

[17] N. Konno Phys. Rev. E 72, 026113 (2005).

[18] M. A. Jafarizadeh and S. Salimi, J. Phys. A: Math. Gen. 39, 13295 (2006) 
[19] C. Moore and A. Russel Quantum walks on the hypercube Proc. 6th Workshop on Randomization and Aproximation Techniques in Computer Science (Lecture Notes in Computer Science 2483) ed J D P Rolim and S Vadhan (Berlin: Spinger) pp 164-78 (arXiv: quant-ph/0104137) (2002).

[20] S. Salimi, Ann. Phys. 324, 1185 (2009).

[21] X. P. Xu, J. Phys. A: Math. Theory. 42, 115205 (2009).
[22] X. Xu, W. Li and F. Liu, Phys. Rev. E 78, 052103 (2008).

[23] P. Lo, S. Rajaram, D. Scheoens, D. Sullivan, C. Tamon and J. Ward, Quantum Inf. Comput. 6, 370-81 (2006).

Yanyan Wang School of Mathematics and statistics , Northwest Normal University, Lanzhou, Gansu, China, Mobile No 17693100584. 